\title{
Performance Analysis of a Novel Double Error Correcting Code for Image Transmission over UWB Channel
}

\author{
Joanne Gomes \\ Research Scholar \\ SNDT University, Mumbai
}

\author{
Dr. B K Mishra \\ Principal \\ Thakur College of Engineering and Technology, Mumbai
}

\begin{abstract}
In the previous correspondence we introduced a binary double error correcting long code $(825)$, for $100 \%$ data correction. This paper evaluates the BER performance of UWB system using (8 2 5) code over UWB (SV) channel for image transmission. The simulation results presented here uses BPSK, as well as 2dimensional Hermite pulse modulation. Image to be transmitted over UWB channel is compressed by using Huffman coding to achieve lossless compression. The simulink model is developed to implement the basic UWB system without any means for channel correction (equalizer). The receiver is simple correlator instead of rake receiver, which is generally used to combat multipath. The performance of the double error correcting code for UWB communication is evaluated here to achieve $100 \%$ accurate image at the receiver.
\end{abstract}

\section{General Terms}

Wireless Communication

\section{Keywords}

Error-Correcting-Code, Generator-Matrix, Image-Compression, UWB-Communication

\section{INTRODUCTION}

Ultra wideband (UWB) is a bandwidth efficient wireless communication technique. In UWB, data is modulated over narrow impulses of nanosecond duration which facilitates high data rate transmission for short distances up to 10 meter, especially in indoor home networking [1][2]. Number of modulation techniques based on Time Hopping Spread Spectrum using Gaussian pulse, for multi-user communication were introduced in [3][4][5][6]. Hermite pulses were first introduced in [7][8] for UWB communication. BER performance of Hermite based modulation techniques have been presented by us in [9] [10]. Faster transfer of multimedia contents from video camera to $\mathrm{PC}$ or a TV in home networking is the need of an hour. Along with the speed of delivery, quality of the received picture is also important. This paper evaluates the performance of (8 25 ) double error correcting code (ECC) for a specific application of image transmission over AWGN and UWB channel in view of improving the accuracy of received data. The error correcting code is an integral part of any communication system to combat the channel noise. Shannon in his work proved that SNR of -1.6 $\mathrm{dB}$ over AWGN channel can achieve BER of $10^{-5}$ at the receiver. Hamming introduced single bit error correcting codes. Golay introduced $(23,12)$ triple error correcting perfect code. Multiple error correction was achieved by $\mathrm{BCH}$ codes. All these and many other codes assumed that the minimum dimension of the code $(\mathrm{k})$ should be three. In [11][12] we introduced a novel and simple binary long code $\left(\begin{array}{ll}8 & 2\end{array}\right.$ ) for double error correction where code length is ' 8 ', code dimension is ' 2 ' and minimum (Hamming) distance of the code is ' 5 '. The code performance is directly proportional to the code length. As per this theorem the code (8 2 5) corrects 2-bit errors at the receiver when two data bits are transmitted thus achieving $100 \%$ data correction, as long as the errors occur in less than or equal to two bits. The encoder/decoder circuits and the interesting properties of this code have been presented in our previous work [12]. Along with accuracy, the speed of data delivery can be improved by Hermite based pulse shape modulation techniques introduced in [7][8][13][14][15].

The image to be transmitted over channel is compressed by using Huffman coding to achieve lossless compression. The aim of this work is to achieve $100 \%$ accurate image at the receiver which will also be useful for highly sensitive applications. The BER simulations are performed over AWGN and UWB (SV) channel CM1, using two types of modulations: BPSK and 2-dimensional Hermite based modulation. The paper is organized as follows.

Section II gives a review of binary double error correcting ( $\left.\begin{array}{lll}8 & 2 & 5\end{array}\right)$ long code. Section III highlights the UWB SV channel and the Hermite modulation for UWB system. Section IV elaborates on source coding for image compression and explains the channel encoder and decoder for channel coding whereas section $\mathrm{V}$ presents the simulink model for UWB transmitter/receiver using BPSK modulation with source and channel coding. Section VI assimilates the simulation results and observations. Section VII lists simulation parameters whereas section VIII makes comparative study. Section IX concludes the presented work and elaborates on future scope.

\section{DOUBLE ERROR CORRECTING CODE}

Let $\mathrm{F}$ be a finite field of order $\mathrm{q}$. A linear code $\mathrm{c}$ of length $\mathrm{n}$ over $\mathrm{F}$ is a vector space $\mathrm{v}$. The minimum distance between two vectors of code $\mathrm{c}$ is equal to $\mathrm{d}(\mathrm{c})=\mathrm{d}$, then a vector space of dimension $\mathrm{k}$ and with the minimum Hamming distance of a code $d(c)=d$, is denoted by $(\mathrm{n} \mathrm{k}$ d). If a code can be constructed with the minimum distance of $2 \mathrm{t}+1$ between two code words, then any number of errors per codeword which does not exceed $t$ can be corrected. As per these rules in [12] we devised a binary double error correcting long code ( 825 ) with $1 / 4$ code rate, which corrects both the transmitted bits at the receiver achieving $100 \%$ data correction. The generator matrix of this code was devised using generator polynomial of GF $\left(2^{6}\right)$ as follows [12][19].

$$
g(x)=\left(1+x^{2}+x^{3}+x^{4}+x^{6}\right)
$$




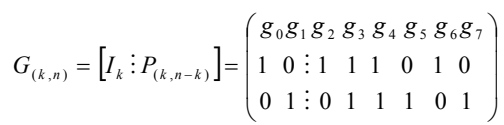

Now parity check matrix is given by

$$
\begin{gathered}
H_{(n-k, n)}=\left\lfloor P^{T}{ }_{(n-k, k)} \vdots I_{n-k}\right\rfloor \\
H_{(n-k, n)}=\left(\begin{array}{c}
10 \vdots 100000 \\
11 \vdots 010000 \\
11 \vdots 001000 \\
01 \vdots 000100 \\
10 \vdots 000010 \\
01 \vdots 000001
\end{array}\right)
\end{gathered}
$$

This code $\left(\begin{array}{lll}8 & 2 & 5\end{array}\right)$ is a systematic cyclic code and its code vectors are

$$
\begin{aligned}
& \mathrm{C} 1=\left[\begin{array}{llllllll}
\mathbf{0} & 0 & 0 & 0 & 0 & 0 & 0 & 0
\end{array}\right] \quad \mathrm{C} 2=\left[\begin{array}{llllllll}
\mathbf{0} & 1 & 0 & 1 & 1 & 1 & 0 & 1
\end{array}\right] \\
& \mathrm{C} 3=\left[\begin{array}{llllllll}
1 & 0 & 1 & 1 & 1 & 0 & 1 & 0
\end{array}\right] \quad \mathrm{C} 4=\left[\begin{array}{llllllll}
1 & 1 & 1 & 0 & 0 & 1 & 1 & 1
\end{array}\right]
\end{aligned}
$$

For this code, total of 36 error vectors (r) per message (single bit error- 8 and double bit error-28) can be received on the receiver side. This will give us 36 unique syndromes $\left(\mathrm{s}=\mathrm{r}^{*} \mathrm{H}^{\mathrm{T}}\right)$ of six bits (n-k) long and will help us to find which one or two bits in the received vector are in error, by using syndrome decoding and look table method.

The Weight distribution $\mathrm{W}(\mathrm{C})$ of an error correcting code $C$, is defined as the set of $n+1$ integers $W(C)=\{A i, 0 \leq \mathrm{i} \leq \mathrm{n}\}$ such that there are $A i$ code words of Hamming weight $i$ in $C$, for $i=0$, $1, \ldots, n$.

The weight distribution of this code is given in the following table-1.

Table 1. Weight distribution of a new code

\begin{tabular}{|c|l|l|l|l|l|l|l|l|l|}
\hline I & 0 & 1 & 2 & 3 & 4 & 5 & 6 & 7 & 8 \\
\hline $\mathrm{Ai}$ & 1 & 8 & 28 & 18 & 27 & 36 & 21 & 8 & 1 \\
\hline
\end{tabular}

Asymptotic coding gain is the gain that would be delivered if vanishingly small decoded error rates were required. It is given by $\mathrm{G}_{\mathrm{a}}=10 \log [\mathrm{R}(\mathrm{t}+1)]$ or $\mathrm{G}_{\mathrm{a}}=10 \log [\mathrm{R} * \mathrm{dmin}]$. Thus asymptotic coding gain for this code will be between -1.249 to $0.969 \mathrm{~dB}$.

This code is very simple and easy to use. Its cyclic nature allows simple implementation of encoder and decoder using shift registers as explained in [12]. Near optimum performance of theoretical code can be achieved with this code using practically simple encoding and decoding procedure. This is done at the cost of transmission bandwidth. Thus the only disadvantage of this code is its low code rate of $1 / 4$ which reduces the data transmission rate. Wideband technologies such as UWB have abundant bandwidth. By using this error correcting method in UWB environment, along with the multidimensional modulation techniques could help us achieve reasonably good transmission rates.

\section{UWB CHANNEL AND HERMITE MODULATION}

IEEE 802.15.3a standard for wireless personal area network specifies UWB channel models (CM1 to CM4) for indoor communication based on the work of Saleh-Valenzuela (SV) [16][17]. The UWB SV channel is frequency selective as shown in fig- 1 and is represented by multiple paths or rays, having real positive gains $\left\{\alpha_{k}\right\}$, propagation delays $\left\{\theta_{k}\right\}$, and associated phase shifts $\left\{\tau_{k}\right\}$, where $k$ is the path index; in principle, $k$ extends from 0 to $\infty$. These rays usually arrive in clusters. If $\mathrm{T}_{l}$ is the arrival time of $1^{\text {th }}$ cluster then for the $\mathrm{k}^{\text {th }}$ ray of the $\mathrm{l}^{\text {th }}$ cluster, the complex, low-pass impulse response of the $\mathrm{S}-\mathrm{V}$ channel1 (CM1) is given by

$$
h(t)=X \sum_{l=0}^{L} \sum_{k=o}^{K} \alpha_{k, l} \delta\left(t-T_{l}-\tau_{k, l}\right)
$$

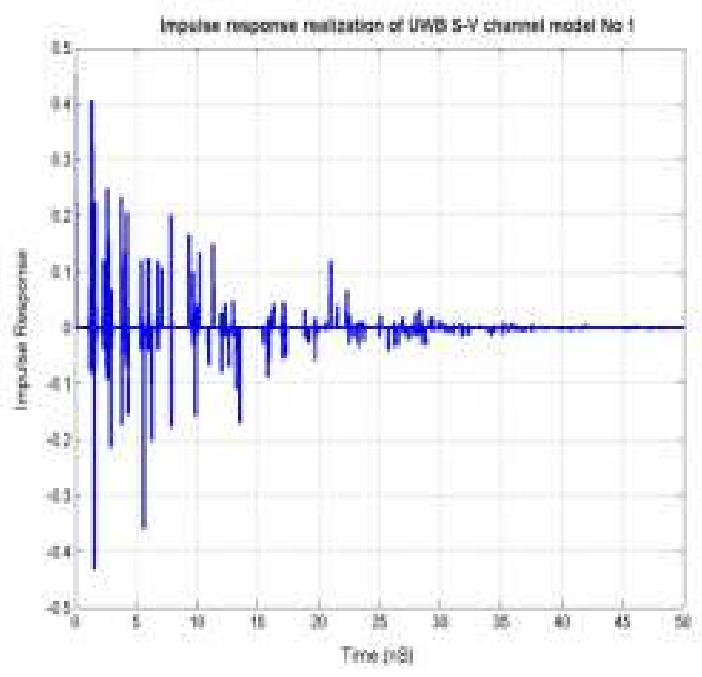

Figure 1. Impulse response of UWB channel (CM1)

In this paper the simulation results are obtained using UWB channel CM1, which is characterized for the line of sight communication over the distance of 4 meter.

Hermite-polynomial based orthogonal pulses, also known as Hermite pulses were proposed in [7] for pulse shape modulation (PSM). Hermite pulses which are smooth functions are well concentrated in both time and frequency domains, ensuring that all the pulses have the same duration. This relates to achievable data-rate while maximizing frequency efficiency. They are orthogonal to one another and individual spectra of Hermite pulses are not identical. Due to these advantages of Hermite pulse over other pulses its use in communication system can help in enhancing data rates by reducing requirement of bandwidth. First order Hermite functions are given by equation- 5 .

$$
\psi_{n}(t)=\frac{H_{n}(t) e^{-t^{2} / 2}}{\sqrt{2^{n} n ! \sqrt{\pi}}}
$$




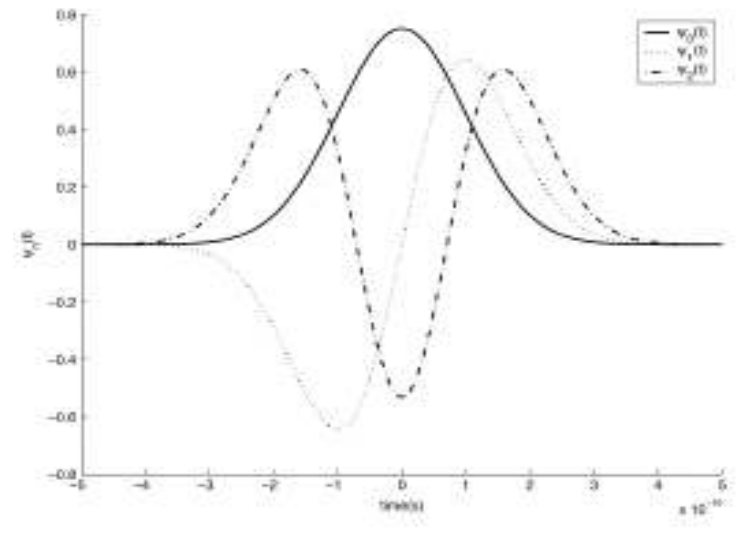

Figure 2. First three Hermite functions

Where the Hermite polynomials, $\mathrm{H}_{\mathrm{n}}(\mathrm{t})$ are recursively obtained by the formula given by equation- 6 . Fig- 2 shows first three Hermite functions and fig-3 shows the spectrum of 0.5 nanosecond Hermite pulse used for UWB communication.

$$
\begin{gathered}
H_{0}(t)=1 \\
H_{1}(t)=2 t \\
H_{2}(t)=4 t^{2}-2
\end{gathered}
$$
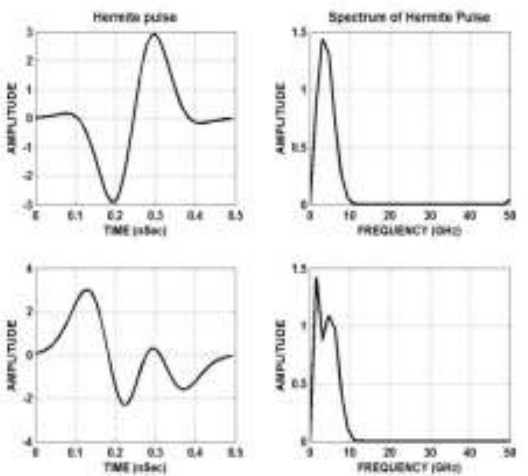

Figure 3. Hermite Pulses and their spectrum

Commonly used modulation techniques in Impulse Radio are PPM, PAM and BPSK along with time-hopping (TH) spread spectrum for multiple access. The simulations performed by us in [9][10] show that BER of PSM is higher than that of PPM or BPSK over UWB (SV) channel. But the advantage of PSM is the increased data rate. In this paper PSM (2-dimensional) and BPSK modulation techniques are used for simulating a basic UWB transmitter-receiver system without any means for channel correction (equalizer). The receiver is simple correlator instead of rake receiver which is generally used to combat multipath.

\section{SOURCE AND CHANNEL CODING}

Source code compresses the source symbols by reducing the redundancy. Lossless image compression involves no loss of data so that the transmitted original source image can be reconstructed at the receiver without any difference. Consider the image of size
$128 \times 128$ (16384) pixels with each pixel taking any value ranging from 0 to 255 . To encode this image into binary code, minimum 8 bits per pixel need to be used giving total 131072 bits. Using Huffman lossless compression the same image is encoded with 124799 bits [20] to achieve the marginal amount of compression. The simulations presented in the paper use the image of size $128 \times 128$ pixels (Lena.jpeg), compressed using Huffman coding.

Channel codes introduce controlled redundancy in the data to be transmitted and are used to protect the data against the channel noise. In this paper the binary double error correction long code explained in section II is used as a channel code. The code has code rate of $1 / 4$. This will reduce the original data transmission rate by factor 4 with BPSK modulation. The UWB system is also simulated here, with 2-dimensional Hermite based modulation. This doubles the data rate at the cost BER.

\section{SIMULINK MODEL FOR UWB SYSTEM}

This section introduces the Simulink model of TH-BPSK UWB system as shown in fig-4. It consists of an image source coder, channel coder and modulator on the transmitter side whereas demodulator and channel decoder on the receiver side. It also consists of error calculation block to simulate the BER performance of the given system. The source coder block takes an image of size $128 \times 128$ pixels that is to be transmitted. This image is compressed by using Huffman coding technique shown in fig- 5 for lossless compression. 'Lena.jpg' image has been used here for which ' 124799 ' bits are required for lossless representation. This image data is coded with double error correcting code explained earlier with the help of linear encoder block of simulink as shown in fig- 6 on the transmitter side and on the receiver side demodulated data is decoded using parity check matrix of the double error correcting code with the help of linear decoder block of simulink as shown in fig-7. BPSK modulator modulates the coded image data over the phase of first order Hermite pulse. This signal is then transmitted over UWB channel. The received image data is demodulated by BPSK demodulator block and then decoded by Linear Binary Decoder block. Decoded image data bits are then uncompressed using Huffman decoder for image reconstruction and then PSNR is calculated between transmitted and received images. This part is not included in the following fig. 4 , since the focus of the work is on performance of the Double error correcting code. Error calculation block is inserted to evaluate the BER performance of the given system. Display will indicate the BER for particular value of EbNo. BER variable to MATLAB workspace is used along with MATLAB BERTool to simulate BER performance of the UWB system.

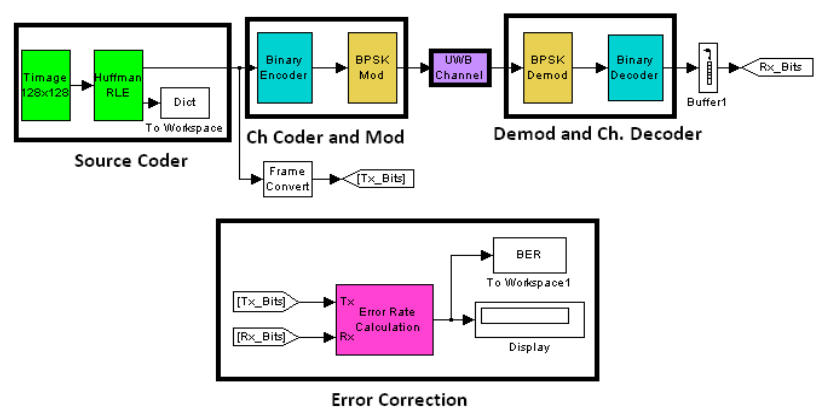

Figure 4. Simulink model of UWB system 


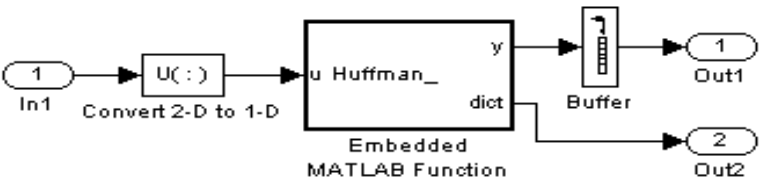

Figure 5. Huffman coding block

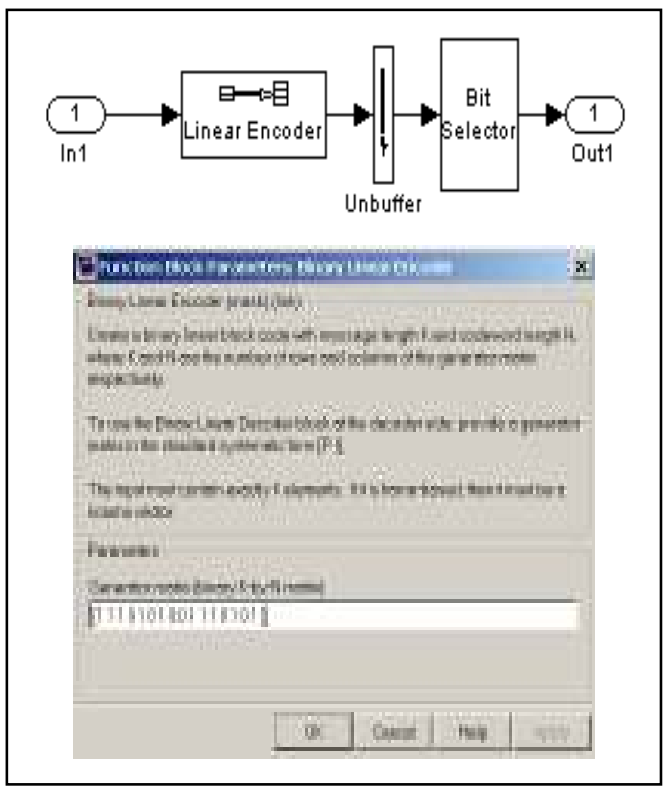

Figure 6. Channel Encoder

UWB channel block is taken from [18] that implements AWGN channel plus UWB multipath (SV) channel CM1 which is originally modeled for Line-of-Sight communication over the distance of 4 meters [16][17]. Its impulse response is indicated in fig-1.
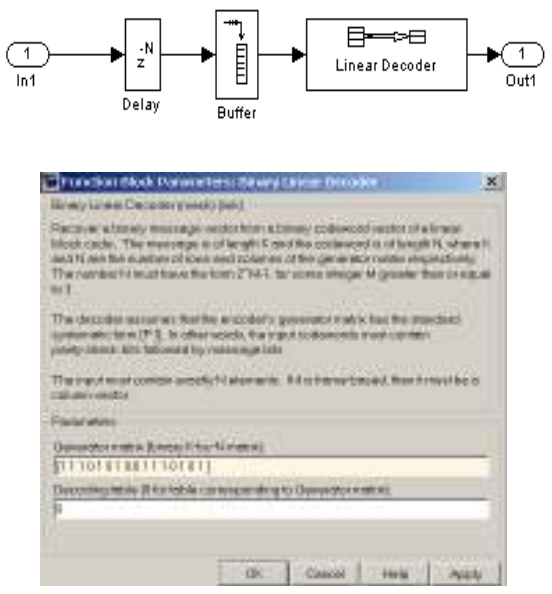

Figure 7. Channel Decoder

\section{SIMULATION PARAMETERS}

The UWB system presented here uses Time-hopping spread spectrum technique as a multiple access technique for three users.
But the simulation results are currently obtained for a single user data transmission. In a Time Hopping (TH) scheme, the symbol duration $T_{s}$ is split into $\mathrm{N}$ frames with 1 pulse per frame [21]. Within each frame, the pulse can take $\mathrm{M}$ equiprobable positions. In addition to modulation, in order to change the shape of the spectrum, data symbols are encoded using pseudorandom (PN) codes introducing time delay in generated pulses, which leads to Time-Hopping UWB. The FCC has set stringent limit on out of band emission mask for UWB communication. Effective Isotropic Radiated Power (EIRP) for UWB range of operating frequencies $(3.1-10.6 \mathrm{GHz})$ is given as EIRP $=\mathrm{Pt} \mathrm{Gt}=-41.3 \mathrm{dBm} / \mathrm{Hz}=74.4$ nanowatts. Where Pt is transmitted power and Gt is transmitting antenna gain. For signal occupying bandwidth of $500 \mathrm{MHz}$, EIRP will be 74.4 nanowatts $x 500=37$ microwatts. $=-14.3 \mathrm{dBm}$.

Considering Hermite pulse of width $\mathrm{Tm}=0.5$ nanosecond for BPSK modulation as shown in fig-3, time hopping frame for one pulse is given by Tframe $=1.5$ nanosecond $(0.5 \times 3)$. Within the frame the pulse has unit energy. There is only one pulse per user per Tframe. Fig- 8 gives two BPSK modulated pulses for bit ' 0 ' and ' 1 respectively. If pulse width $=\mathrm{t}=0.5 \mathrm{~ns}$, chip time $=\mathrm{Tc}=$ $1 \mathrm{~ns}, \mathrm{k}=$ bit 0 or $1, \mathrm{Cj}=$ random time hopping code (e.g. $0,1,2$ etc) with cardinality, $\mathrm{N}=$ number of frames/symbol then using $\psi_{n}(t)$ of equation-5 as the carrier pulse [20], TH-BPSK signal is given by

$$
S_{k}(t)= \pm \sum_{j=o}^{N} \Psi(t-j * T c * N h-C j * T c)
$$

Fig-9 shows two Tframes with two different Hermite pulses for pulse shape modulation of 2-dimension. Here each pulse phase represents two bits $(00,01,10,11)$ thus doubling the data transmission rate. The following equation 8 represents the THPSM signal that is transmitted for bit 0 .

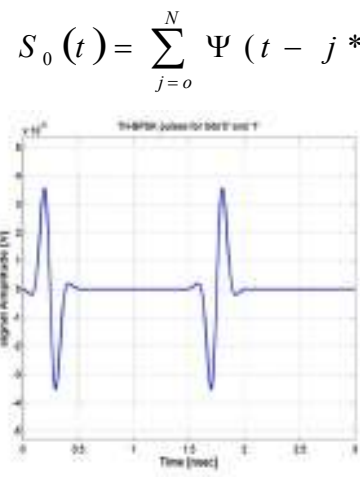

Figure 8. BPSK pulses
The table- 2 below summarizes the simulation parameters set for UWB system represented by fig- 4 .

Table 2. UWB System Parameters

\begin{tabular}{|l|l|}
\hline Model Parameters & Values \\
\hline Pulse energy & Normalized to unity \\
\hline Pulse width & 0.5 nanoseconds \\
\hline TH-frame width & 1.5 nanoseconds \\
\hline Bit rate without coding & $1 / 1.5$ nanosecond \\
\hline
\end{tabular}




\begin{tabular}{|l|l|}
\hline (with coding) & $\begin{array}{l}\sim 667 \mathrm{MBps}(167 \mathrm{MBps}) \text {---BPSK } \\
\sim 1334 \mathrm{MBps}(334 \mathrm{MBps}) \text {-PSM }\end{array}$ \\
\hline Image compression & Lossless \\
\hline $\begin{array}{l}\text { Channel coding rate with } \\
(82 \text { 5) long code }\end{array}$ & $1 / 4$ \\
\hline Modulation & BPSK, PSM (2-Dimensional) \\
\hline $\begin{array}{l}\text { Number of pulses / } \\
\text { symbol }\end{array}$ & One \\
\hline UWB channel & CM1 parameters \\
\hline Equalization & Nil \\
\hline
\end{tabular}

\section{RESULTS and OBSERVATIONS}

To perform BER simulations the UWB system of fig-4, modeled in simulink is used. Hermite pulse of unit energy is BPSK modulated and transmitted over AWGN as well as over UWB channel. A simple correlator [22] is used as a receiver without any channel estimation. Fig-10 shows BER of BPSK with and without ( $\left.\begin{array}{lll}8 & 2 & 5\end{array}\right)$ error correcting code over AWGN and UWB channel along with theoretical BER of BPSK. Fig-11 shows the quality of received images with PSNR equal to '14.7' and ' 45.65 ' when they were transmitted using BPSK modulation without ECC over AWGN channel, with SNR (EbNo) of '7' and ' 8 ' $\mathrm{dB}$ respectively. With BPSK without ECC over AWGN, $\mathrm{SNR}=10 \mathrm{~dB}$ is required to receive image with $100 \%$ accuracy i.e. with $P S N R=$ infinity. Over UWB channel, SNR of $15 \mathrm{~dB}$ is required for BPSK to achieve $100 \%$ accuracy in image transmission. If $(825)$ ECC is used with BPSK over AWGN then required SNR reduces by 5 to $6 \mathrm{~dB}$ to achieve the same BER performance. Fig-12 shows quality of received images over AWGN with ECC. $100 \%$ accurate image is received at $\mathrm{SNR}=4 \mathrm{~dB}$. BPSK with $\mathrm{ECC}$ over $\mathrm{UWB}$ channel requires higher SNR to receive $100 \%$ accurate image. This is due to ISI at the receiver as an effect of multipath UWB channel.

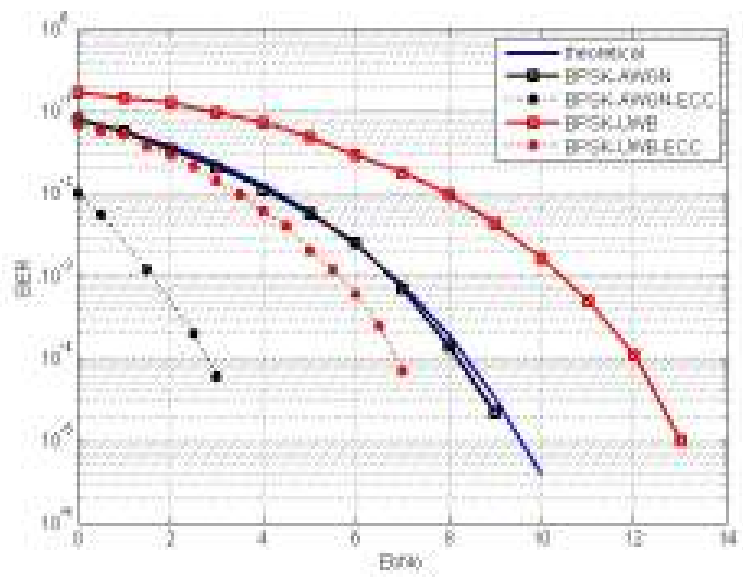

Figure 10. BER of BPSK over AWGN and UWB

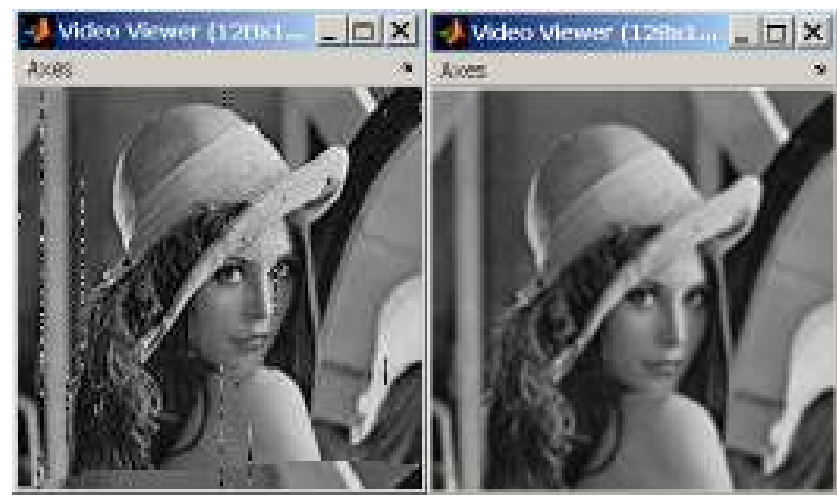

Figure 11. $B P S K S N R=7, P S N R=14,7 \& S N R=8, P S N R=45.65$

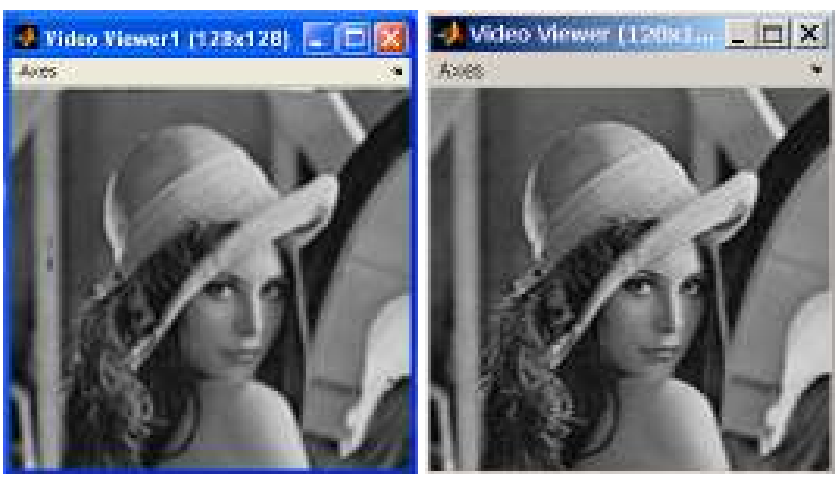

Figure 12. $B P S K E C C S N R=1 P S N R=22.9, S N R=4, P S N R=$ inf

To achieve better results over UWB channel, one needs to estimate and equalize the channel effects. Similar simulations were carried out using pulse shape modulation (PSM) with and without ECC over AWGN and UWB multipath channel. PSM has originally high bit error rate than BPSK due to the fact that orthogonal Hermite pulses tend to lose their orthogonality over frequency selective channel such as UWB. This reduces the correlation between two pulses resulting in the increased BER. Fig-13 gives BER of PSM with two pulses (2-dimensional) over AWGN and UWB channel. Fig-14 represents the quality of received images with different SNR using PSM+ECC technique.

Table 3. Simulation Results

\begin{tabular}{|c|c|c|c|c|}
\hline $\begin{array}{l}\text { Modulation } \\
\text { Technique }\end{array}$ & $\begin{array}{c}\text { EbNo } \\
\text { dB }\end{array}$ & BER & $\begin{array}{l}\text { PSNR of } \\
\text { Image }\end{array}$ & $\begin{array}{c}\text { Data Rate } \\
\text { Mbps }\end{array}$ \\
\hline \multirow{3}{*}{$\begin{array}{c}\text { BPSK } \\
(\mathrm{AWGN})\end{array}$} & 7 & $\sim 10 \mathrm{E}-3$ & 14.7 & \multirow[t]{3}{*}{667} \\
\hline & 8 & $10 \mathrm{E}-4$ & 45.65 & \\
\hline & 10 & $\sim 0$ & infinity & \\
\hline \multirow{2}{*}{$\begin{array}{c}\text { BPSK } \\
(\mathrm{AWGN}) \\
\text { with }\left(\begin{array}{ll}8 & 25\end{array}\right) \\
\mathrm{ECC}, \mathrm{R}=1 / 4\end{array}$} & 1 & $\sim 10 \mathrm{E}-3$ & 22.9 & \multirow[t]{2}{*}{166} \\
\hline & 4 & $\sim 0$ & infinity & \\
\hline \multirow{3}{*}{$\begin{array}{l}\text { BPSK } \\
\text { (UWB) }\end{array}$} & 11 & $\sim 10 \mathrm{E}-4$ & 40 & \multirow[t]{3}{*}{667} \\
\hline & 13 & $10 \mathrm{E}-5$ & 78 & \\
\hline & 15 & $\sim 0$ & infinity & \\
\hline BPSK (UWB) & 6 & $10 \mathrm{E}-3$ & 30 & 166 \\
\hline
\end{tabular}




\begin{tabular}{|c|c|c|c|c|}
\hline \multirow{2}{*}{ 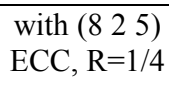 } & 7 & $\sim 10 \mathrm{E}-5$ & 56 & \\
\hline & 9 & $\sim 0$ & infinity & \\
\hline & & & & \\
\hline \multirow{3}{*}{$\begin{array}{l}\text { 2-D-PSM } \\
(\mathrm{AWGN})\end{array}$} & 10 & $10 \mathrm{E}-3$ & 20 & \multirow[t]{3}{*}{1334} \\
\hline & 13 & $10 \mathrm{E}-5$ & 82 & \\
\hline & 14 & $\sim 0$ & infinity & \\
\hline \multirow{3}{*}{$\begin{array}{c}\text { 2-D-PSM } \\
(\mathrm{AWGN}) \\
\text { with }\left(\begin{array}{ll}8 & 25\end{array}\right) \\
\mathrm{ECC}, \mathrm{R}=1 / 4\end{array}$} & 6 & $\sim 10 \mathrm{E}-4$ & 37 & \multirow[t]{3}{*}{332} \\
\hline & 7 & $\sim 10 \mathrm{E}-5$ & 49 & \\
\hline & 9 & $\sim 0$ & infinity & \\
\hline \multirow{3}{*}{$\begin{array}{c}\text { 2-D-PSM } \\
\text { (UWB) }\end{array}$} & 16 & $\sim 10 \mathrm{E}-4$ & 45 & \multirow[t]{3}{*}{1334} \\
\hline & 18 & $10 \mathrm{E}-5$ & 77 & \\
\hline & 20 & $\sim 0$ & infinity & \\
\hline \multirow{3}{*}{$\begin{array}{c}\text { 2-D-PSM } \\
\text { (UWB) } \\
\left.\text { with ( } \begin{array}{ll}8 & 25\end{array}\right) \\
\text { ECC, R=1/4 }\end{array}$} & 12 & $10 \mathrm{E}-4$ & 39 & \multirow[t]{3}{*}{332} \\
\hline & 13 & $\sim 10 \mathrm{E}-5$ & 53 & \\
\hline & 15 & $\sim 0$ & infinity & \\
\hline
\end{tabular}

\section{COMPARATIVE STUDY}

The following figures 15 and 16 referred from references [14] and [15] show the BER of pulse shape modulation technique using Hermite pulses of different types for UWB communication. From the figures we observe that BER of 10 E-4 is achieved at SNR varying from 13 to $15 \mathrm{~dB}$.

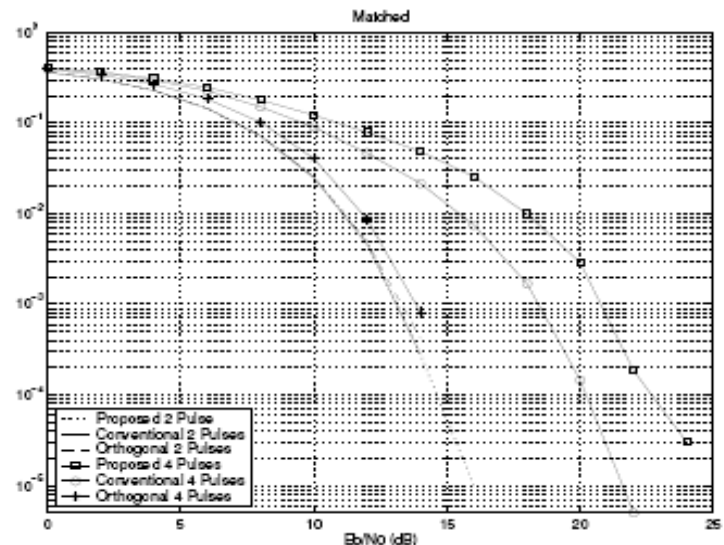

Figure 15. BER of PSM-UWB Systems with Proposed and Conventional pulses [14]

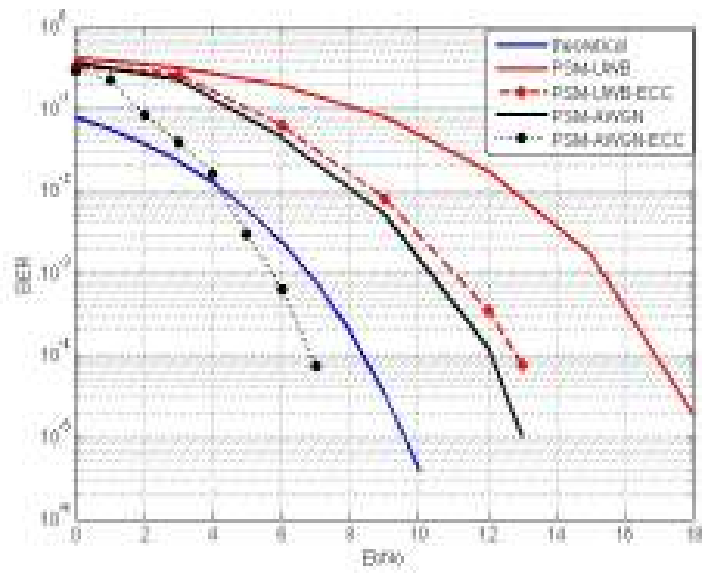

Figure 13. BER of PSM (2-D) over AWGN and UWB

Table-3 summarises the simulation results obtained in this paper. It lists BER and PSNR at different SNRs and gives approximate data rates that could be achieved with different schemes.

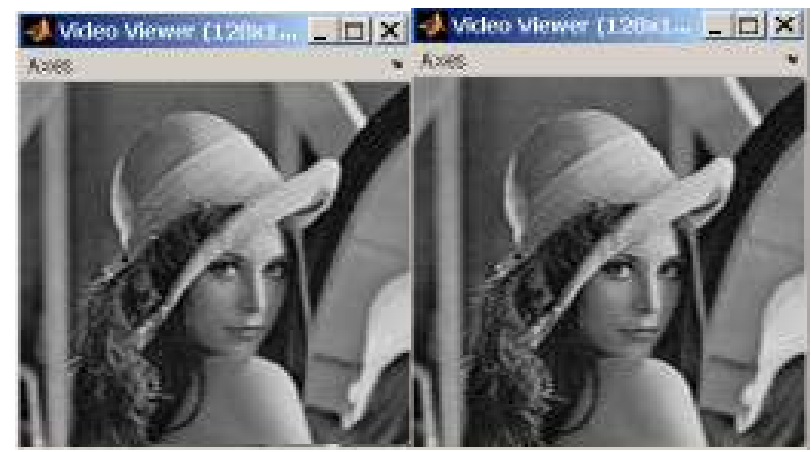

Figure 14. $\mathrm{PSMECC} \mathrm{SNR}=6 \mathrm{PSNR}=27.3 \& \mathrm{SNR}=7 \mathrm{PSNR}=58$

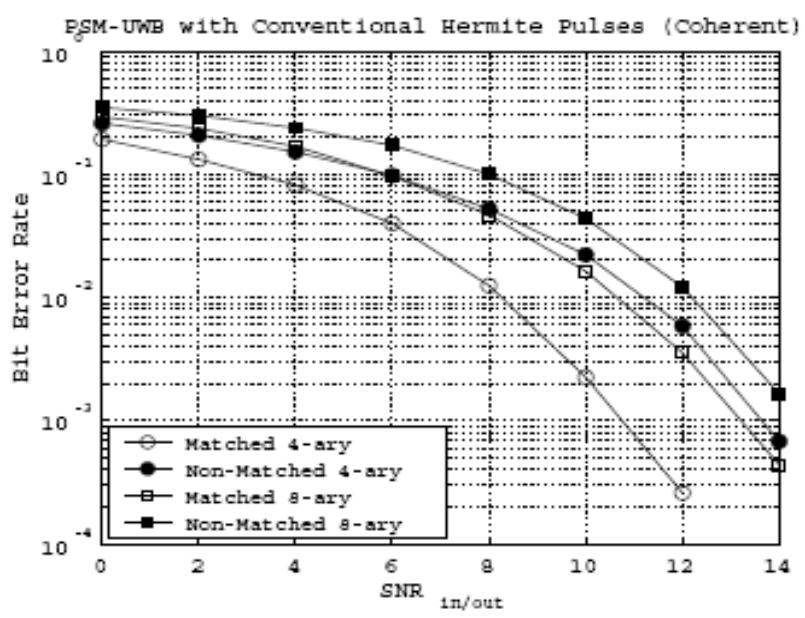

Figure 16. BER of 4-ary and 8-ary PSM-UWB system [15]

Comparing these figures- 15 and 16 with figures- 10 and 13, it can be said that the work presented in this paper achieved approximately similar results using basic first order pulses in its original form and simplest error correcting code. The results obtained in this paper using ( $\left.\begin{array}{l}8 \\ 8\end{array} 25\right)$ ECC are the best optimum results in the given situation. These results can be improved to great extent by using the remedies for maintaining the orthogonality of the pulses and by reducing the multipath effect of the UWB channel with the help of equalizer.

\section{CONCLUSION}

The paper evaluated the performance of binary $\left(\begin{array}{lll}8 & 2 & 5\end{array}\right)$ double error correcting code (with $100 \%$ data correction capability) for UWB communication, for a specific application of image transmission. The aim was set to receive the transmitted image with $100 \%$ accuracy at the receiver end, over UWB channel. 
It is observed that ( $\left.\begin{array}{lll}8 & 2 & 5\end{array}\right)$ ECC improves the UWB system and makes it energy efficient by reducing required SNR, by the factor of 5 to $6 \mathrm{~dB}$ to achieve reasonably good BER. Over UWB channel BPSK with ECC requires just 9dB SNR whereas PSM requires $15 \mathrm{~dB}$ SNR to achieve BER of 10E-5 and image PSNR of infinity. The UWB multipath channel has been used here without any channel estimation or equalization. Hence the results obtained here are the best optimum results in the given situation. These results can be improved to great extent by using the remedies for maintaining the orthogonality of the Hermite pulses and by reducing the multipath effect of UWB channel with the help of equalizer. This will be the future scope of the work.

\section{REFERENCES}

[1] M Z. Win and R. A. Scholtz. 1993 Impulse Radio: How it Works. In IEEE Communications Letters, vol. 2, 36-38.

[2] Roberto Aiello and G D Rogerson. 2003 Ultra-Wideband Wireless Systems. In IEEE microwave magazine, 628-635.

[3] J. A. N. da Silva and M. L. R. de Campos, "Performance comparison of binary and quaternary UWB modulation schemes," Proc. Globecom, vol 2, 2003, pp 789-793.

[4] Jeju, Korea. Marcel D. Blech, Daniel Geier, and Thomas F. Eibert. 2008 Concept of an UWB Impulse Radio B-/QPSK Transmitter Based on Standard Logic Components. In Proceedings of IEEE International Conference On UltraWideband (ICUWB2008), Vol. 1, 225-228.

[5] M. Z. Win and R. A. Scholtz. 2000 Ultra-wide bandwidth time-hopping spread-spectrum impulse radio for wireless multiple-access communications. In IEEE Trans Commun., vol. 48, 679-689.

[6] R A Scholtz, 1993 Multiple Access with Time Hopping Impulse Modulation. In Millitary Commun. Conference, IEEE vol. 2, 447-450.

[7] J. A. N. da Silva and M. L. R. de Campos. 2002 Orthogonal Pulse Shape Modulation for Impulse radio. In IEEE Proc. Of the International Telecommunications Symposium, 916-921.

[8] M. Ghavami, L. B. Michael, and R. Kohno' "Hermite function based orthogonal pulses for UWB communications," Proc. WPMC, Denmark, 2001, pp 437440 .

[9] J Gomes and B K Mishra. 2009 Orthogonal Hermite Pulses for Indoor Communication with Ultra Wideband channel. In Proceeding of International Conference on Computational Intelligence, comm., Systems and network, IEEE Xplore.
[10] J Gomes and B k Mishra. 2010 Multidimensional Hermite based Modulation with Dual Error Correction over UWB Channel. In ACM Proceedings of the International conference ICWET, Mumbai 362-367.

[11] J Gomes and B k Mishra. 2010 A New Double Error Correcting Long Code For 100\% Correction. In Springer Proceedings of the International Conference on Contours of Computing Technology Mumbai 276-279.

[12] J Gomes and B K Mishra. 2010 Double Error Correcting Long Code. In AIRCC- IJCNC, International Journal of Computer Networks and Communications Sept 2010.

[13] C. J. Mitchell, G. T. F. de Abreu and R. Kohno. 2002 Two Dimensional Codulation for UWB Systems. In IEICE Proc. of the Symposium on Information Theory and Applications, Japan.

[14] G. T. F. de Abreu, C. J. Mitchell, L. G. F. Trichard, and R. Kohno. 2003 A note on the application of hermite pulses in uwb communications. In Proc. IEEE 1st International Workshop on Ultra Wideband Systems (IWUWBS'03), Oulu, Finland.

[15] G. T. F. de Abreu, C. J. Mitchell, and R.Kohno. 2003 On the design of orthogonal pulse-shape modulation for UWB systems using Hermite pulses. in J. Commun. NetworksSpecial Issue on Ultra-Wideband Commun., vol. 5, 328-343.

[16] Adel A M Saleh and Reinaldo A Valenzuela. 1987 A Statistical Model for Indoor Multipath Propagation. In IEEE Journal on Selected Areas in Commun., vol.2, 128-137

[17] D. Cassioli, M. Z. Win, and A. F. Molisch. 2002 The ultrawide bandwidth indoor channel from bandwidth indoor channel: from statistical model to simulations. In IEEE Journal Select. Areas Commun, vol 20(6), 1247-1257.

[18] Martin Clark, Mike Mulligan, Dave Jackson, and Darel Linebarger. 2004 Fixed-Point Modeling in an Ultra Wideband (UWB) Wireless Communication System, MATLAB Digest - May 2004.

[19] S. Lin and D.J. Costello. 1983 Error Control Coding: fundamentals and applications, Prentice Hall.

[20] Khalid Sayood. 2001 Data Compression. Second edition, Elsevier.

[21] Maria Gabriella and Guerino Giancola. 2002 Understanding Ultra Wide Band Fundamentals. First edition, Prentice Hall.

[22] Proakis. 2001 Digital Communication. Ffourth edition, MC Graw Hill. 